\title{
Gold nanoparticle trapping and delivery for therapeutic applications
}

This article was published in the following Dove Press journal:

International Journal of Nanomedicine

28 December 201I

Number of times this article has been viewed

\section{MS Aziz'}

Nathaporn Suwanpayak ${ }^{3,4}$

Muhammad Arif Jalil ${ }^{2}$

R Jomtarak ${ }^{4}$

T Saktioto ${ }^{2}$

Jalil Ali'

PP Yupapin ${ }^{4}$

Institute of Advanced Photonics Science, ${ }^{2}$ lbnu Sina Institute of

Fundamental Science Studies, Nanotechnology Research Alliance, Universiti Teknologi Malaysia, Johor Bahru, Malaysia; ${ }^{3}$ King Mongkut's Institute of Technology Ladkrabang, Chump on Campus, Chumphon, ${ }^{4}$ Nanoscale Science and Engineering Research Alliance (N'SERA), Faculty of Science, King Mongkut's Institute of Technology Ladkrabang, Bangkok, Thailand
Correspondence: PP Yupapin Nanoscale Science and Engineering Research Alliance (N'SERA), Advanced Research Center for Photonics, Faculty of Science, King Mongkut's Institute of Technology Ladkrabang, Bangkok 10520, Thailand

Tel +66 23298414

Fax +6623298410

Email kypreech@kmitl.ac.th; isoqt@kmitl.ac.th
Abstract: A new optical trapping design to transport gold nanoparticles using a PANDA ring resonator system is proposed. Intense optical fields in the form of dark solitons controlled by Gaussian pulses are used to trap and transport nanoscopic volumes of matter to the desired destination via an optical waveguide. Theoretically, the gradient and scattering forces are responsible for this trapping phenomenon, where in practice such systems can be fabricated and a thin-film device formed on the specific artificial medical materials, for instance, an artificial bone. The dynamic behavior of the tweezers can be tuned by controlling the optical pulse input power and parameters of the ring resonator system. Different trap sizes can be generated to trap different gold nanoparticles sizes, which is useful for gold nanoparticle therapy. In this paper, we have shown the utility of gold nanoparticle trapping and delivery for therapy, which may be useful for cosmetic therapy and related applications.

Keywords: gold nanoparticle trapping, particle trapping, therapy, transport

\section{Introduction}

The interaction of nanoparticles with biological systems, ie, living cells, has become an attractive field of research in material science and biology. It holds the key for the advent of bionanotechnology, which is important for the interaction and manipulation of biological samples at the nanoscale. Gold nanoparticles are widely used for biological application and medical purposes due to their unique optical properties and electrochemical stability. ${ }^{1-3}$ The properties of gold nanoparticles are such that when light interacts with matter, the light is absorbed, scattered at the same frequency as the incoming light, reemitted, and any spectroscopic signals from the molecules at material surface is enhanced. ${ }^{4}$ Further, the gold nanoparticle surface is facile. Specifically, various biomolecules can be conjugated to gold particles directly with surface functionalization, ${ }^{5}$ and are nontoxic and nonimmunogenic. ${ }^{6}$ Gold nanoparticles can also be used as a carrier for delivering molecules into cells. ${ }^{7,8}$ The trapping phenomenon of the gold nanoparticles occurs in the Rayleigh region, ${ }^{9}$ in which the diameter of the sample is much smaller than the trapping beam wavelength. ${ }^{10}$ The optical trapping mechanism arises from the fact that light exerts force when it interacts with matter and this phenomenon is known as optical tweezers. ${ }^{11}$ This is an important technique with diverse applications in communications and biology. Recently, optical tweezers have been used widely to investigate the increment numbers in biochemical and biophysical processes, ${ }^{12}$ cell interactions, cell manipulation without breaking the membrane, and to measure adhesion force between cells. ${ }^{13-15}$ 
The use of optical trapping tools within an add-drop multiplexer for microscopic volume trapping and delivery has been reported in both theoretical and experimental science. ${ }^{16,17}$ The carrier signal works in the same way as the optical tweezers interact with microparticles in a noninvasive manner. The phenomenon of static tweezers has been well analyzed, and the use of dynamic tweezers is an emerging field due to their wide applications. ${ }^{18,19}$ Schulzyz et al have demonstrated the transfer of trapped atoms between two optical potentials ${ }^{20}$ based on the principle that a highly focused laser beam exerts a gradient and scattering force that can be used to trap and move microscopic volumes of matter. In this paper, the PANDA ring resonator, an optical trapping tool, traps and transports molecules or atoms by using dark soliton pulses as input and Gaussian pulses to control the signal propagating within an add-drop optical multiplexer integrated with two nanorings on the left and right sides. The carrier signals in the form of optical vortices or potential well can be transported as dynamic optical tweezers. These are highly stable signals with no fluctuation over a certain period of time so that the atom or molecule does not disappear during the delivery process. ${ }^{21}$ The desired dynamics of the optical tweezers can be achieved (by providing suitable input power and controlling the signals within the system), tuned, and stored within the ring resonator system before it reaches the desired destination via an optical waveguide. By using the proposed system, gold nanoparticles can be trapped, transported, and delivered. The proposed system can be used in biological and medical applications for diagnostic, therapeutics, imaging, and optical-sensing purposes.

\section{Theory}

The utility of optical tweezers are based on the force light exerts on a matter when it interacts with it. The basic principle in optical tweezers is that the momentum transfer is associated with bending light as the quanta of light ${ }^{22}$ carries energy proportional to its momentum and in the direction of propagation. ${ }^{23}$ When light passes through an object, it refracts and changes direction. ${ }^{24}$ This causes a change in the momentum of the light. ${ }^{25}$ To conserve the total momentum, an object acquires momentum equal to that lost by the photons. This shows that a number of forces act on the object, which is known as radiation pressure (Figure 1). The sum of forces can be split into two components, ie, the scattering force, in the direction of incident light and the gradient force, arises from the intensity gradient pointing towards the center of the beam. ${ }^{26,27}$ These two forces can be used to hold and move microscopic dielectric objects physically. In application, the

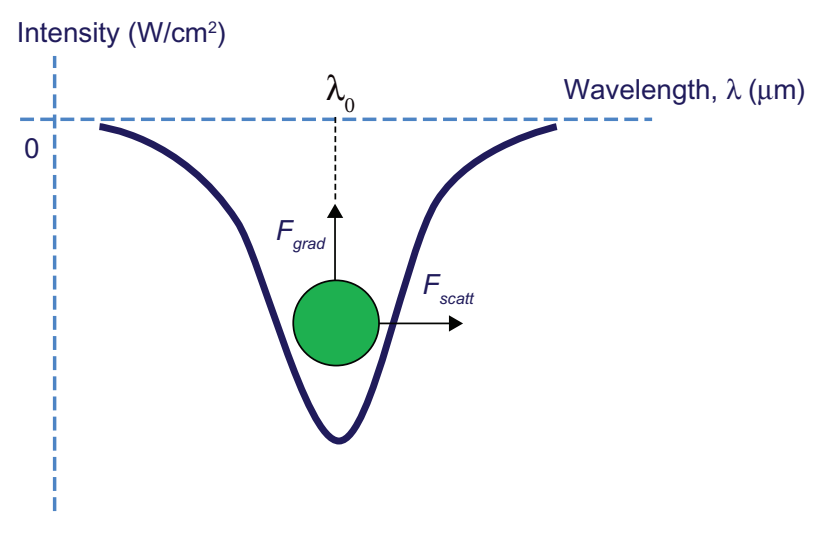

Figure I Basic principles of optical tweezers.

Note: A particle is trapped in the potential well formed by dark solitons at the center wavelength, $\lambda_{0}$.

radiation pressure from a focused laser beam is able to trap and manipulate microscopic objects as small as a single atom. ${ }^{13}$

This trapping phenomenon can be categorized into two types as Rayleigh trapping and Mie trapping. If the size (d) of the trapped particle is much smaller than the wavelength of the trapping beam $(\mathrm{d}<<\lambda)$, such trapping is known as trapping in the Rayleigh regime. In this case, wave optics are required to explain the force acting on the particles. Particle can be considered as a miniscule dipole immersed in the optical trapping field oscillating at frequency $v$. The two forces acting on the particles are known as dipole scattering and Lorentz force (gradient force). In Mie trapping, the size of the trapped particle is larger than the wavelength $(d>>\lambda)$. In this case, the electric field is not uniform and geometrical optics are used to describe the forces acting on the system. In this paper, the Rayleigh trapping is described in detail because the gold nanoparticle diameters are much smaller than the wavelength of the carrier signals. The optical forces that act on the trapped particles can be defined as: ${ }^{28}$

$$
F=\frac{Q n_{m} P}{c}
$$

$Q$ represents the fraction of power utilized to exert force, which is equal to 1 , known as dimensionless efficiency, $n_{m}$ is the refractive index of the suspending medium, $P$ is the incident laser power, measured at the tested sample or volume and $c$ is the speed of light. For plane wave incident on a perfectly absorbing particle to achieve stable trapping, the radiation pressure must create a stable and three-dimensional equilibrium. As biological specimens are usually immersed in an aqueous medium, the dependence of force $\mathrm{F}$ on $n_{m}$ can be used to achieve higher trapping forces. Increasing the laser power is possible, but only over a limited range due to 
the possibility of optical damage. $Q$ is therefore the main determinant of trapping force and depends upon the numerical aperture (NA), laser wavelength, light polarization state, laser mode structure, relative index of refraction, and particle geometry. The scattering force is given as:

$$
F_{\text {scatt }}=n_{m} \frac{\langle S\rangle \sigma}{c},
$$

The scattering force is proportional to the energy flux and points along the direction of propagation of the incident light. The gradient field $\left(F_{\text {grad }}\right)$ is the Lorentz force acting on the dipole induced by the light field. It is given as:

$$
F_{\text {grad }}=\frac{\alpha}{2} \nabla\left\langle E^{2}\right\rangle
$$

The large gradient force is formed by the large depth of the laser beam. Stable trapping requires that the gradient force is in the $-\hat{z}$ direction against the direction of incident light (dark soliton valley). By increasing the NA and decreasing the focal spot size, the gradient strength can be increased. ${ }^{29,30}$ This occurs within a tiny system, for instance, a nanoscale device such as a nanoring resonator.

In this paper, dark soliton is used as a carrier and Gaussian pulse as a control signal propagating within the PANDA ring resonator. The potential well is formed between the gaps produced by two forces to confine atoms or molecules. The generated optical tweezer can propagate within the optical waveguide, thus trapped particles can be transported to the desired destination. ${ }^{31}$ The dynamics of the optical tweezers are tuned and controlled by optimizing the parameters and input power of the system. $E_{L}$ and $E_{R}$ are the fields that propagate within the nanorings on the left and right hand sides of the add-drop filter. $\mathrm{E}_{\text {in }}$ and $\mathrm{E}_{\text {add }}$ represent the input and control optical fields fed into the system. ${ }^{21}$

\section{Gold nanoparticles}

Various force spectroscopy techniques are used to investigate nanoscopic systems, but optical tweezers are the most flexible because of their unique ability to precisely measure the three-dimensional motion of particles from small molecules to whole living cells. This technique provides fine control over a wide range of applied forces on the sample, which is important especially for biological specimens. In operation, the dark soliton as carrier signal is used to trap and store nanoparticles within the PANDA ring resonator system. Optical tweezers can propagate within optical waveguides, hence the micro- and nanoscopic volume (particles) can be transported from one point to another. ${ }^{32,33}$ In this paper, we propose the use of optical trapping tools for trapping, transporting, and delivering gold nanoparticles within optical waveguides. Gold nanoparticles can be used to probe a particular nanoscale system noninvasively. For the study of biological systems, it is customary to attach gold beads to the system of interest; the nanometer-scale motion and the forces acting on the bead can then be monitored. The gold nanoparticle size ranges from 5 to $100 \mathrm{~nm}$, which is well synthesized and characterized..$^{8,34}$ Gold nanoparticles can be trapped and transported within the proposed system.

Generally, gold particles are difficult to trap due to the strong scattering and absorption forces. For stable threedimensional trapping, gradient forces must be stronger than the scattering and absorption forces acting on the particle. Small gold nanoparticles are more suitable for trapping and delivery. This is because scattering and absorption forces decrease faster than the gradient force with particle diameter. ${ }^{35}$ The maximum force that could be exerted on the particles in the lateral direction was found using Stokes' drag calibration. For particles ranging from $101 \mathrm{~nm}$ to $254 \mathrm{~nm}$ diameter, the maximum force was found to be in the range of $0.56 \mathrm{pN}$ to $2.2 \mathrm{pN}$ using a laser power of $135 \mathrm{~mW}$ (the larger the particle, the larger the maximum force). Thus, the maximum force can exert into the proposed system by using the full laser power of $7 \mathrm{~W}$, which will be around 20 $\mathrm{pN}$. Normally, $20 \mathrm{pN}$ of force is sufficient enough to hold and manipulate cells or nanoparticles. ${ }^{28,33}$ The term opticution refers to the interaction between a very-high-flux laser light $\left(10^{7} \mathrm{~W} / \mathrm{cm}^{2}\right)$ and biological sample by breaking covalent bonds and causing photochemical reaction, this might cause cell damage or death. ${ }^{36}$ In this research, the range of values will not cause opticution on the biological sample, thus it can be used in investigations of nanoscale systems where the force range is important. In simulation, dark soliton is generated at center wavelength $1.6 \mu \mathrm{m}$ with peak power

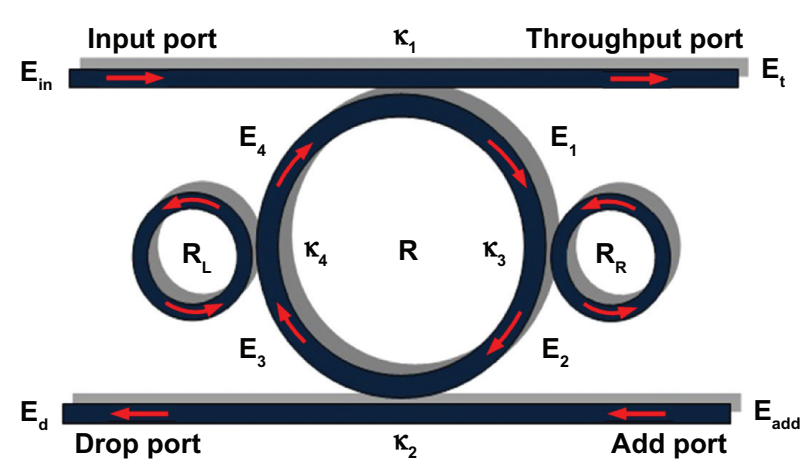

Figure 2 PANDA ring resonator. 
$5 \mathrm{w}$ and pulse width $1.20 \mathrm{~ns}$ fed into the system via an input port. A Gaussian pulse as a control signal is introduced into the system via the add port. The radius of center, left, and right rings are $37 \mu \mathrm{m}, 100 \mathrm{~nm}$, and $100 \mathrm{~nm}$, respectively (Figure 2). The coupling coefficients of the PANDA ring resonator system are set to be $\kappa_{1}=0.16, \kappa_{2}=0.66, \kappa_{3}=0.10$, and $\kappa_{4}=0.10$. The effective core area of the center ring, $A_{\text {effr }}$, equals $4 \mu \mathrm{m}^{2}$ while the effective core areas, $A_{\text {eff }}$, for the right and left side of the center ring (nanoring) are $1 \mu \mathrm{m}^{2}$. The waveguide losses coefficient, $\alpha$ is 0.25 and coupling loss, $\gamma$ is assumed to be 0.01 .

Dynamic tweezers are generated at four different center wavelengths which are 1.4, 1.5, 1.6, and $1.7 \mu \mathrm{m}$ (Figure 3).
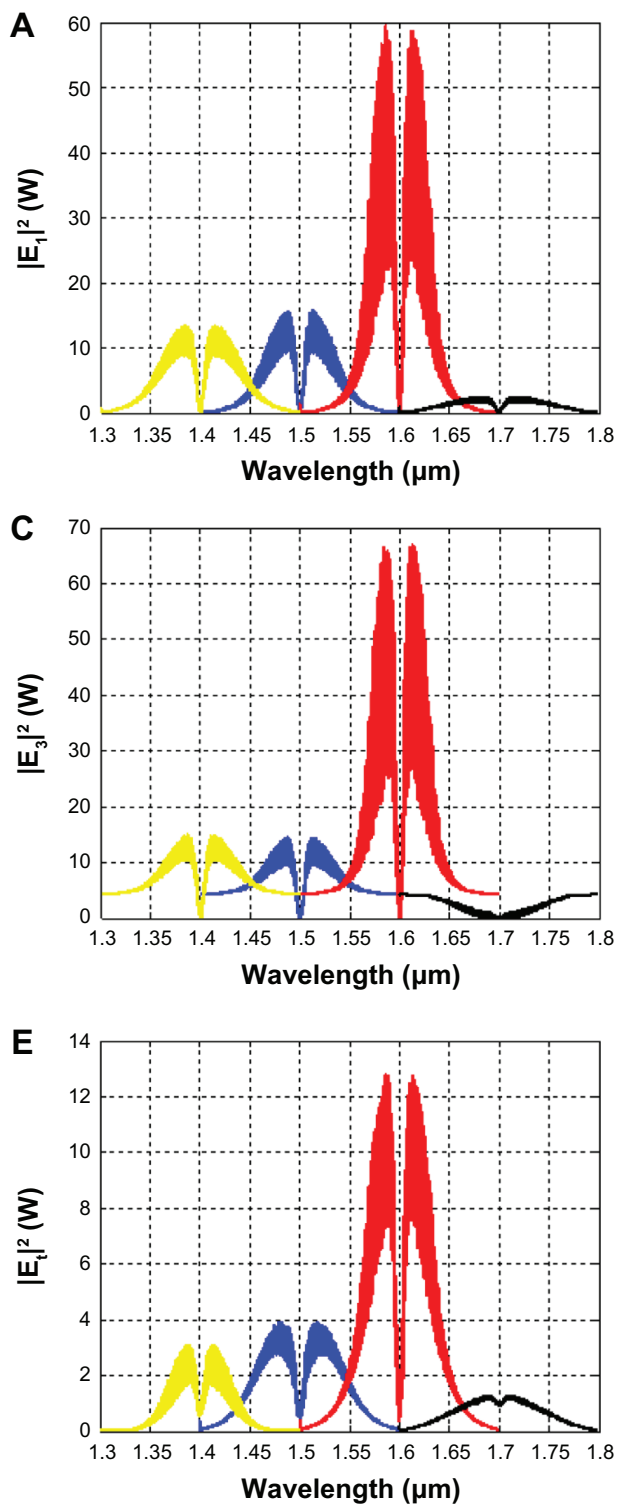

The optical fields circulate within the center ring resonator. $\mathrm{E}_{\mathrm{t}}$ and $\mathrm{E}_{\mathrm{d}}$ are output fields at the throughput and drop ports, respectively. The shape and peak power of the fields are different for each center wavelength used. Based on the generated results, tweezers with the center wavelength of $1.6 \mu \mathrm{m}$ produce the highest intensities at the drop port $(80 \mathrm{~W})$. This shows that gold nanoparticles are stably trapped inside the potential well and can be transported by optical tweezers signals. The important aspect of this simulation is that the required dynamic behavior of the tweezers can be obtained by tuning some parameters of the system including addport input signal, coupling coefficient, and ring radius. By controlling these parameters, the required number of gold
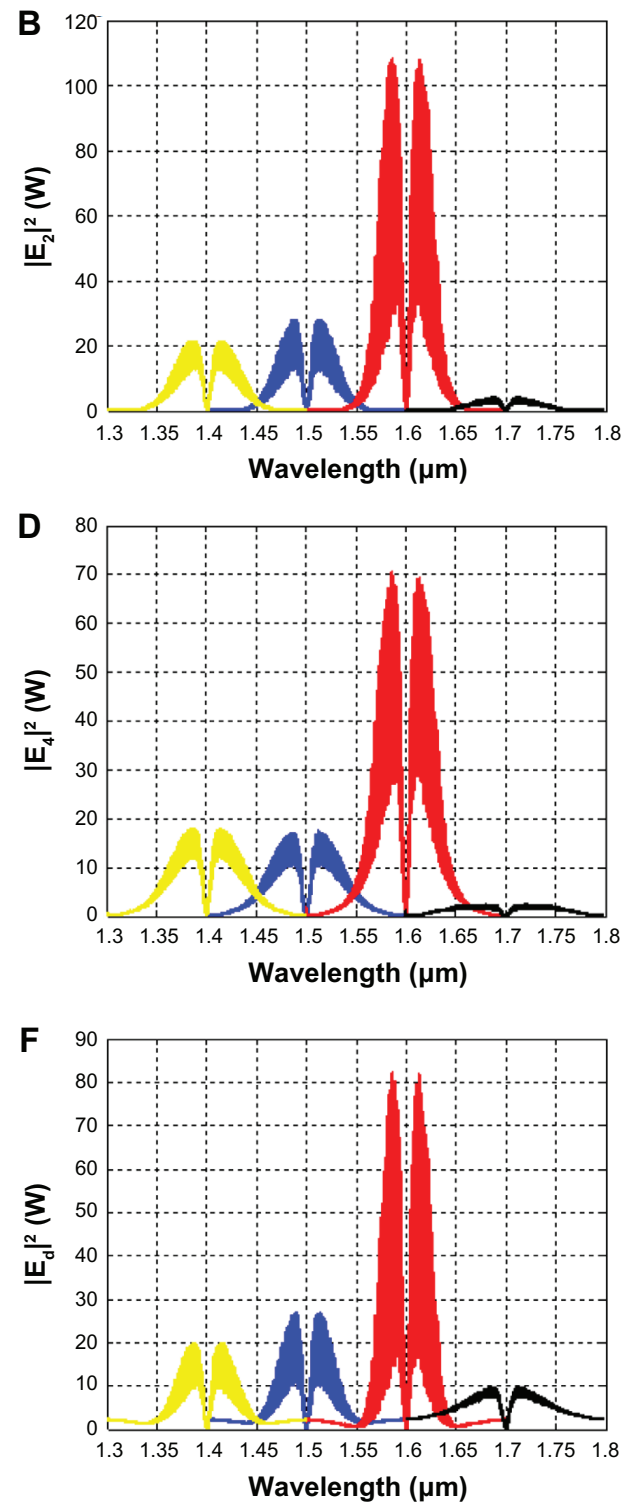

Figure 3 Results of dynamic optical tweezers generated at four different center wavelengths, where $E_{1}, E_{2}, E_{3}$, and $E_{4}$ are circulated in optical fields within the center ring resonator shown in (A), (B), (C), and (D), respectively. $E_{t}$ and $E_{d}$ are output optical fields generated at the throughput and drop ports of the PANDA ring as shown in (E) and (F), respectively. 

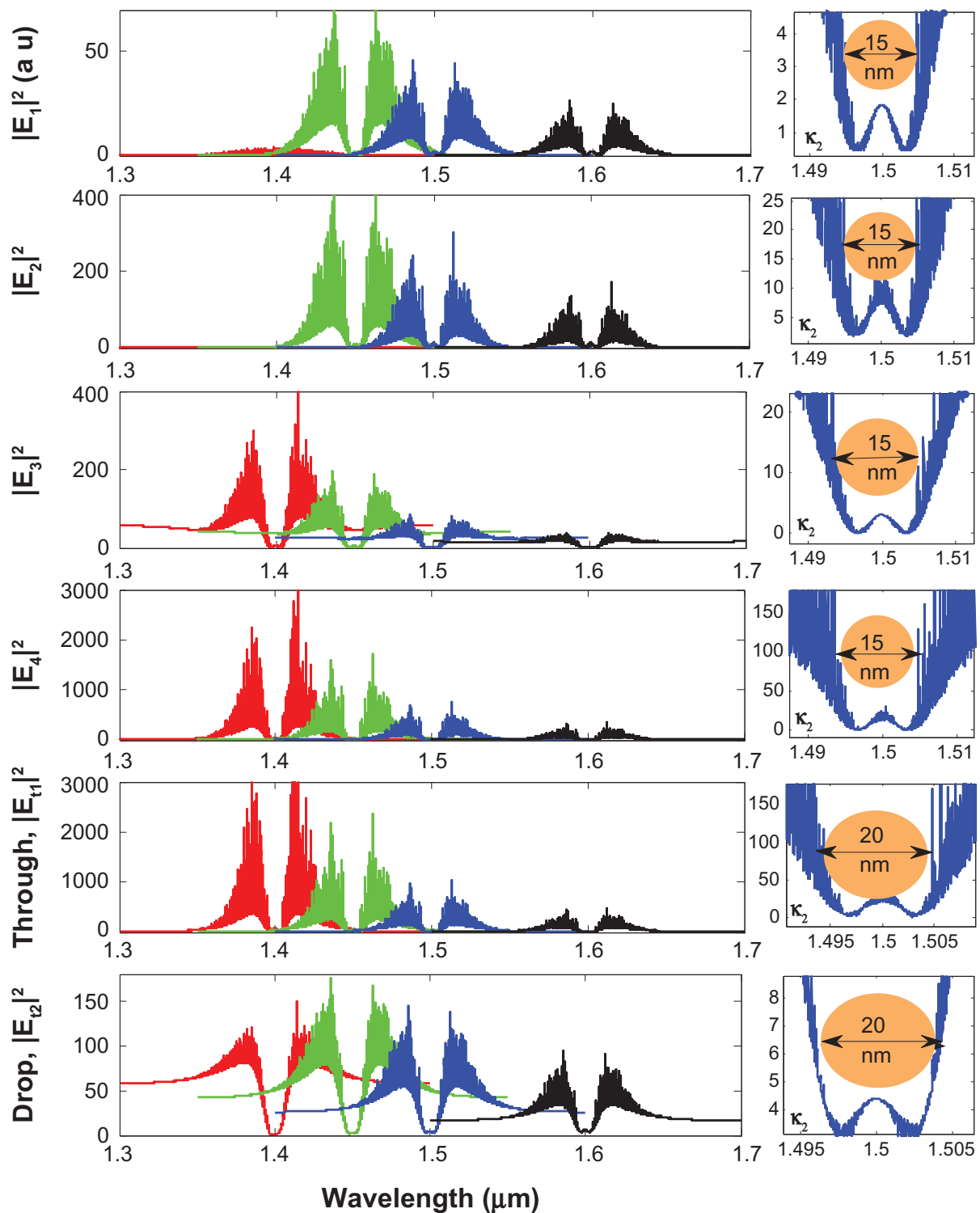

Wavelength $(\mu \mathrm{m})$

Figure 4 Results of the optical trapping tool with different wavelength.

Notes: Add-drop filter radius $=10 \mu \mathrm{m}$; right-hand ring radius and left-hand ring radius $=4 \mu \mathrm{m}$.

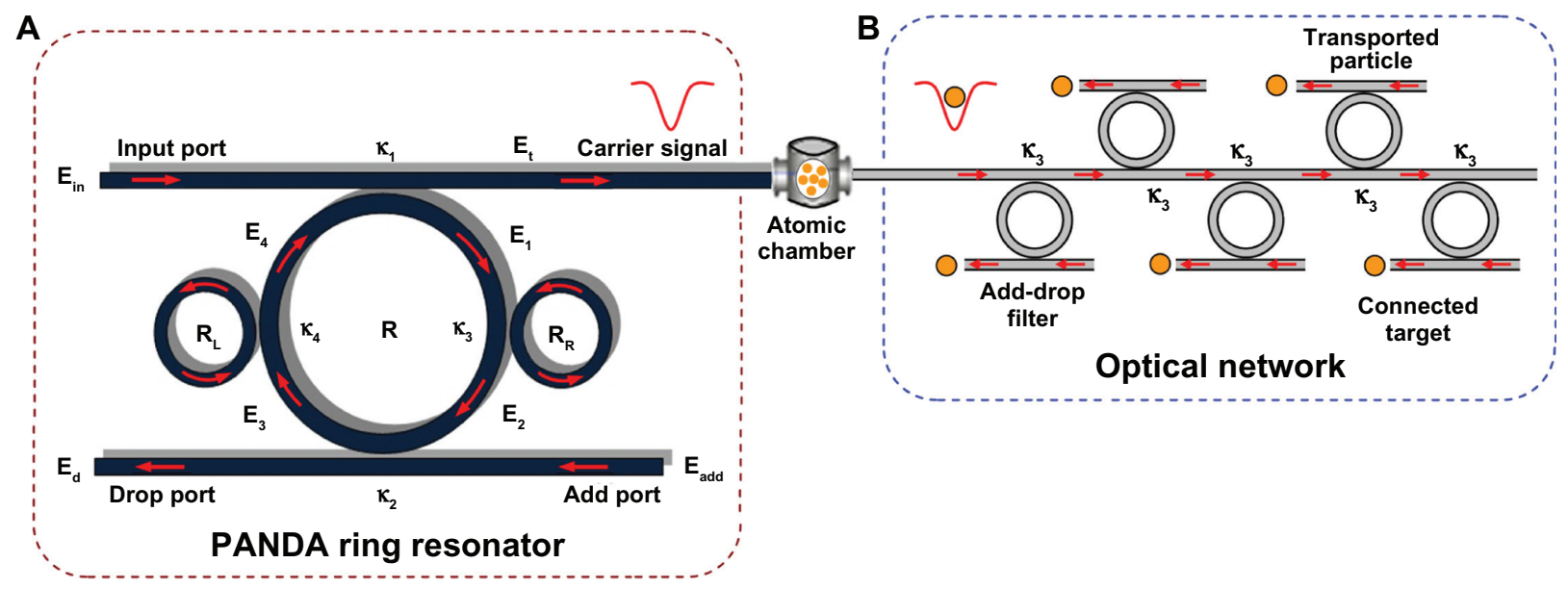

Figure 5 Proposed system for trapping and transporting gold nanoparticles to the required target. A shows trapping part, B shows transporting part. Abbreviations: RL, left-hand ring resonator; RR, right-hand ring resonator; $R$, add-drop filter. 
nanoparticles can trapped and transported through throughput and drop ports, otherwise the signal will propagate within the ring before collapsing or decaying into the waveguide. The output of optical tweezers from throughput and drop ports with different ring radii was simulated (Figure 4). Different sizes of trapping are generated, which allows the capture of different gold nanoparticle sizes. In application, the optical tweezers signals can propagate within an optical waveguide, thus demonstrating that the gold nanoparticle can be transported to the required destination by using the optical fiber. The proposed system uses trapping signals in the form of dark solitons that can be converted to bright solitons when passed through an add-drop filter with specific parameters, and the gold nanoparticles are safely retrieved by the receiver at drop port of the system (Figure 5). ${ }^{16,32}$ In the proposed system, we used the spherical gold nanoparticle to conjugate molecular therapy which has excellent enhanced Raman scattering surface properties ${ }^{37}$ and are known to be nontoxic and nonimmunogenic. ${ }^{38}$ The gold particles can be trapped and transported via the suitable waveguide to the required targets or destinations, which can be useful for the applications such as atom bottom-up, drug delivery, cosmetics, and nanoelectronic devices.

\section{Conclusion}

Our results show that gold nanoparticles can be trapped and transported via an optical waveguide by using dynamic optical tweezers generated from dark soliton as a carrier signal and Gaussian pulse as control signal. The dynamic behavior of the optical tweezers can be controlled by tuning the optical pulse input power and parameters of the PANDA ring resonator system. Nanorings on both sides of the resonator amplify the potential well and as a result the stability of the trapping signals increases. The use of a molecular buffer and resonator system to build the long-distance gold nanoparticle trapping and delivery can be realized by using the proposed system. The trapped gold nanoparticles can be transported via an optical waveguide to the required target, where users can retrieve the desired signal (messages). The problem of trapping atoms or molecules with different sizes can be solved by using tunable optical tweezers where the depths of the potential well can be adjusted to the required size. This tunable tweezing technique can be used primarily in nanomedicine applications such as drug trapping, delivery, storage (molecular buffer), and delivery via a quantum router with the addition of the PANDA ring resonator for amplification purposes, and blood cleaning in artificial kidney and human artificial hearts. Due to its smart structure (on-chip design), low cost, and long-lasting design, the proposed system is of great importance in the near future.

\section{Acknowledgments}

We would like to thank the Institute of Advanced Photonics Science, Nanotechnology Research Alliance, Universiti Teknologi Malaysia (UTM) and King Mongkut's Institute of Technology (KMITL), Thailand for providing research facilities. This research work has been supported by UTM's Tier 1/Flagship Research Grant, MyBrain15 Fellowship/ MOHE SLAB Fellowship, and the Ministry of Higher Education (MOHE) research grant.

\section{Disclosure}

The authors declare no conflicts of interest in relation to this work.

\section{References}

1. Pellegrino T, Sperling RA, Alivisatos AP, Parak WJ. Gel electrophoresis of gold-DNA nanoconjugates. J Biomed Biotech. 2007;2007:26796.

2. Yu MX, Zhou C, Liu JB, Hankins JD, Zheng J. Luminescent gold nanoparticles with $\mathrm{pH}$-dependent membrane adsorption. $J$ Am Chem Soc. 2011;133(29):11014-11017.

3. Yang AH, Moore SD, Schmidt BS, Klug M, Lipson M, Erickson D. Optical manipulation of nanoparticles and biomolecules in sub-wavelength slot waveguides. Nature. 2009;457(7225):71-75.

4. Sperling RA, Rivera Gil P, Zhang F, Zanella M, Parak WJ. Biological applications of gold nanoparticles. Chem Soc Rev. 2008;37(9): 1896-1908

5. Huang X, Jain PK, El-Sayed IH, El-Sayed MA. Gold nanoparticles: interesting optical properties and recent applications in cancer diagnostics and therapy. Nanomedicine (Lond). 2007;2(5):681-693.

6. Han G, Ghosh P, Rotello VM. Multi-functional gold nanoparticles for drug delivery bio-applications of nanoparticles. Adv Exp Med Biol. 2007;620:48-56.

7. Jain PK, Lee KS, El-Sayed IH, El-Sayed MA. Calculated absorption and scattering properties of gold nanoparticles of different size, shape, and composition: Applications in biological imaging and biomedicine. J Phys Chem B. 2006;110(14):7238-7248.

8. Seol Y, Carpenter AE, Perkins TT. Gold nanoparticles: enhanced optical trapping and sensitivity coupled with significant heating. Opt Lett. 2006;31(16):2429-2431.

9. Srinivas P, Patra CR, Bhattacharya S, Mukhopadhyay D. Cytotoxicity of naphthoquinones and their capacity to generate reactive oxygen species is quenched when conjugated with gold nanoparticles. Int $J$ Nanomedicine. 2011;6:2113-2122.

10. Svoboda K, Block SM. Optical trapping of metallic Rayleigh particles. Opt Lett. 1994;19(13):930-932.

11. Ashkin A, Dziedzic JM, Bjorkholm JE, Chu S. Observation of a single-beam gradient force optical trap for dielectric particles. Opt Lett. 1986;11(5):288-290.

12. Chen L, Henein G, Luciani V. Nanofabrication techniques for controlled drug-release devices. Nanomedicine (Lond). 2010;6(1):1-6.

13. Ashkin A, Dziedzic JM, Yamane T. Optical trapping and manipulation of single cells using infrared laser beams. Nature. 1987;330(6150): 769-771.

14. Chen HD, Ge KK, Li YM, et al. Application of optical tweezers in the research of molecular interaction between lymphocyte function associated antigen-1 and its monoclonal antibody. Cell Mol Immunol. 2007;4(3):221-225. 
15. Seeger S, Monajembashi S, Hutter KJ, Futterman G, Wolfrum J, Greulich KO. Application of laser optical tweezers in immunology and molecular genetics. Cytometry. 1991;12(6):497-504.

16. Piyatamrong B, Kulsirirat K, Mitatha S, Yupapin PP. Dynamic potential well generation and control using double resonators incorporating in an add/drop filter. Mod Phys Lett B. 2010;24:3071-3082.

17. Cai H, Poon AW. Optical manipulation and transport of microparticles on silicon nitride microring-resonator-based add-drop devices. Opt Lett. 2010;35(17):2855-2857.

18. Egashira K, Terasaki A, Kondow T. Photon-trap spectroscopy applied to molecules adsorbed on a solid surface: probing with a standing wave versus a propagating wave. Appl Opt. 2010;49(7):1151-1157.

19. Kachynski AV, Kuzmin AN, Pudavar HE, Kaputa DS, Cartwright AN, Prasad PN. Measurement of optical trapping forces by use of the twophoton-excited fluorescence of microspheres. Opt Lett. 2003;28(23): 2288-2290.

20. Schulzyz M, Crepaz H, Schmidt-Kaler F, Eschner J, Blatt R. Transfer of trapped atoms between two optical tweezer potentials. J Modern Opt. 2007;54(11):1619-1626.

21. Teeka C, Jalil MA, Yupapin PP, Ali J. Novel tunable dynamic tweezers using dark-bright soliton collision control in an optical add/drop filter. IEEE Trans Nanobioscience. 2010;9(4):258-262.

22. Wu S, Plisson T, Brown RC, Phillips WD, Porto JV. Multiphoton magnetooptical trap. Phys Rev Lett. 2009;103(17):173003.

23. Grange W, Husale S, Guntherodt H-J, Hegner M. Optical tweezers system measuring the change in light momentum flux. Rev Sci Instrum. 2002;73(6):2308-2316.

24. Moffitt JR, Chemla YR, Izhaky D, Bustamante C. Differential detection of dual traps improves the spatial resolution of optical tweezers. Proc Natl Acad Sci U S A. 2006;103(24):9006-9011.

25. Sheetz MP, Wilson L, Matsudaira P, Laser Tweezers in Cell Biology. San Diego, CA: Academic Press; San Diego, 1997.

26. Ashkin A. Forces of a single-beam gradient force on a dielectric sphere in the ray of optic regime. Biophys J. 1992;61(21):569-582.
27. Ashkin A, Schutze K, Dziedzic JM, Euteneuer U, Schliwa M. Force generation of organelle transport measured in vivo by an infrared laser trap. Nature. 1990;348(6299):346-348.

28. Svoboda K, Block SM. Biological applications of optical forces. Ann Rev Biophy Biomol Struct. 1994;23:247-282.

29. Bauer WR, Nadler W. Molecular transport through channels and pores: Effects of in-channel interactions and blocking. Proc Natl Acad Sci US A. 2006;103(31):11446-11451.

30. Rosenberry MA, Reyes JP, Tupa D, Gay TJ. Radiation trapping in rubidium optical pumping at low buffer-gas pressures. Phys Rev A. 2007;75:023401-1-6.

31. Jalil MA, Tasakorn M, Suwanpayak N, Ali J, Yupapin PP. Nanoscopic volume trapping and transportation using a PANDA ring resonator for drug delivery. IEEE Trans Nanobioscience. 2011;10(2):106-112.

32. Jalil MA, Piyatamrong B, Mitatha S, Ali J, Yupapin PP. Molecular transporter generation for quantum-molecular transmission via an optical transmission line. Nano Communication Networks. 2010; 1(2): 96-101.

33. Suwanpayak N, Jalil MA, Aziz MS, Ali J, Yupapin PP. Molecular buffer using a PANDA ring resonator for drug delivery use. Int $J$ Nanomedicine. 2011;6:575-580.

34. Haiss W, Thanh NTK, Aveyard J, Fernig DG. Determination of size and concentration of gold nanoparticles from UV-Vis spectra. Anal Chem. 2007;79(11):4215-4221.

35. Wang K, Schonbrun E, Crozier KB. Propulsion of gold nanoparticles with surface plasmon polaritons: evidence of enhanced optical force from near-field coupling between gold particle and gold film. Nano Lett. 2009;9(7):2623-2629.

36. Volpe G, Padgett MJ, Molloy J, McGloin D, Optical tweezers: methods and applications. Contemporary Physics. 2011

37. Cai W, Gao T, Hong H, Sun J. Applications of gold nanoparticles in cancer nanotechnology. Int J Nanomedicine. 2008;1:17-32.

38. Brown SD, Nativo P, Smith J-A, et al. Gold nanoparticles for the improved anticancer drug delivery of the active component of oxaliplatin. J Am Chem Soc. 2010;132(13):4678-4684.
International Journal of Nanomedicine

\section{Publish your work in this journal}

The International Journal of Nanomedicine is an international, peerreviewed journal focusing on the application of nanotechnology in diagnostics, therapeutics, and drug delivery systems throughout the biomedical field. This journal is indexed on PubMed Central,

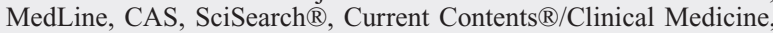

\section{Dovepress}

Journal Citation Reports/Science Edition, EMBase, Scopus and the Elsevier Bibliographic databases. The manuscript management system is completely online and includes a very quick and fair peer-review system, which is all easy to use. Visit http://www.dovepress.com/ testimonials.php to read real quotes from published authors. 